\title{
Comparative effectiveness studies in multiple sclerosis
}

\author{
Carrie M Hersh*,1 \& Le H Hua ${ }^{1}$ \\ ${ }^{1}$ Mellen Program for Multiple Sclerosis, Cleveland Clinic Lou Ruvo Center for Brain Health, Las Vegas, NV 89106, USA \\ *Author for correspondence: hershc@ccf.org
}

\begin{abstract}
"Despite the pragmatic utility of observational studies, they are often prone to confounding by indication, and thus require methods to limit baseline imbalances in demographics and disease characteristics before direct comparisons are made."
\end{abstract}

First draft submitted: 27 March 2020; Accepted for publication: 31 March 2020; Published online: 5 June 2020

Keywords: comparative effectiveness studies $\bullet$ disease modifying therapies $\bullet$ multiple sclerosis $\bullet$ observational studies $\bullet$ propensity score methodology

Multiple sclerosis (MS) is a chronic, autoimmune-mediated, demyelinating and neurodegenerative disease of the $\mathrm{CNS}$ that is the number one cause of nontraumatic disability in young persons. Disease-modifying therapies (DMTs) dramatically improve outcomes by reducing disease activity and progression in MS. However, the emerging DMT landscape remains complex, and as new therapies with variable efficacy and safety profiles become available, these complexities will become even more multifaceted, yielding greater challenges in DMT decision-making in clinical practice [1]. While randomized clinical trials (RCTs) provide the highest level of evidence for DMT safety and efficacy, they are costly, time-prohibitive, and have more limited applications in the clinical setting due to restrictive inclusion criteria. In contrast, observational (nonrandom) studies harnessing real-world data are cost-effective and time efficient, and allow direct comparisons of DMTs in larger, more heterogeneous patient populations to answer clinically relevant questions with broad applicability [2].

\section{Harnessing insights from real-world observational data}

Real-world comparative effectiveness studies evaluating the safety and efficacy of DMTs is a rapidly advancing area of MS clinical research. Harnessing insights from real-world data has gained growing interest in recent years and will continue to expand as the number of novel MS therapies rapidly increase. Well-designed comparative effectiveness studies in MS contribute robust real-world evidence to facilitate decision-making in clinical practice [2] and can also provide useful insights into the effectiveness of DMTs on sub-populations of interest that are not well-represented in RCTs (e.g., older patients, minority populations and those with certain comorbidities).

Observational studies, however, are susceptible to many types of biases, and therefore prone to scrutiny by regulatory agencies and the medical community. While RCTs eliminate biases through the random nature of treatment assignment to prespecified cohorts, observational studies are confounded by multiple biases that challenge their reliability and reproducibility. Such biases include: attrition, arising from between-group differences in followup duration, selection bias, arising from sub-populations being preferentially included in a study, immortal time bias, arising from systematic differences in the definitions of study entry, Will Rogers phenomenon, wherein diagnostic criteria are changed during the study period and indication bias, arising from subjects' exposure to nonrandom treatment [2].

In retrospective observational studies, indication bias can occur when a patient's characteristics that determine treatment selection are also associated with the treatment outcome. Providers choose treatments based on clinical judgment or 'insider information'. For example, a patient who is considered higher risk may receive a more aggressive DMT than a patient who is considered lower risk and started on a less aggressive therapy. However, the higher risk patient may have inherently poorer outcomes compared with the lower risk patient, thus appearing that the aggressive treatment does not work as well as the less aggressive treatment. In this context, individuals who receive 
one DMT might be different from those treated with another DMT, a matter of 'apples' versus 'oranges'. This is in contrast to RCTs where treatment is randomly assigned without considering individual patient factors. In this observational context, treatment groups differ systematically and are therefore noncomparable. Therefore, one must account for such systematic differences in baseline characteristics between groups of interest when estimating the effect of treatment on desired outcomes.

\section{Propensity score analysis for mitigating limitations of observational studies}

Historically, researchers have relied on the use of regression adjustment to account for differences in measured baseline characteristics between treatment groups. However, there has been increasing interest in methods based on the propensity score (PS) [3] to reduce or eliminate the effects of confounding and certain biases (e.g., indication bias) when using observational data. The PS is the probability of treatment assignment based on a prespecified selection of baseline covariates that affect treatment selection, in other words, the various 'insider information' that providers use as a basis of their clinical judgment. PS methodology addresses indication bias by balancing the distribution of covariates across treatment groups before direct comparisons are made. A robust PS model includes a complete list of covariates that are relevant to treatment selection/allocation and are typically prognostic of treatment outcomes. For example, when building a PS model for an MS study evaluating treatment A versus treatment B, important variables to incorporate into the model include demographics (e.g., age, sex and race), baseline disease characteristics (e.g., prior relapses and gadolinium-enhancing lesions, disease course, disease duration, prior number and type of DMTs) and comorbidities (e.g., vascular comorbidities, such as hypertension, hyperlipidemia, diabetes mellitus, tobacco exposure, chronic heart and lung disease). Because the PS is a function of covariates rather than outcomes, the estimated effect is therefore not biased by the desired outcome. PS methods are therefore a useful tool in MS comparative effectiveness studies to enable robust comparisons between treatment groups, approaching that of a randomized study design. In this context, comparisons of treatment groups begin to look more like 'apples' versus 'apples'.

There are various approaches to PS analyses, including matching, weighting and stratification [3,4]. The most common implementation of PS matching is one-to-one or pair matching, in which pairs of treated and untreated subjects are formed, such that matched subjects have similar values of the PS. Thus, in a set of subjects with the same PS, the distribution of observed baseline covariates between treated and untreated groups is the same. This method allows for comparisons between individuals with a similar probability (PS) of receiving the same treatment but in fact received different treatments, thereby mimicking randomization. However, one notable limitation of PS matching is that if the groups of interest have different sample sizes, patients may be unmatched and therefore excluded from the analysis, potentially introducing selection bias. A second approach of PS analysis, inverse probability of treatment weighting, uses weights based on the PS to create a synthetic sample in which the distribution of measured baseline covariates is independent of treatment assignment. A third approach, stratification on the PS involves stratifying subjects into mutually exclusive subsets based on their estimated PS. Within each PS stratum, treated and untreated subjects will have approximately similar values of the PS. Therefore, when the PS has been correctly specified, the distribution of measured baseline covariates will be roughly similar between treated and untreated subjects within the same stratum $[3,4]$.

However, PS methodologies can only adjust for known sources of bias. In the context of 'hidden bias', certain methods can be used to estimate the minimum effect size of an unmeasured covariate to determine if the apparent treatment effect differences were actually attributable to other confounders. For example, the Rosenbaum bounds/Hodges-Lehmann $\Gamma$ quantifies the robustness of the outcome measurement based on hypothetical unmeasured covariates. This sensitivity analysis identifies the proportion of hidden bias that would nullify a statistically significant finding, though it does not identify the specific unmeasured variables [5].

\section{Comparative effectiveness studies for evaluating DMTs in MS}

Several recent, noteworthy comparative effectiveness studies, many of which applied PS methodologies, contributed to the MS literature by reporting on real-world treatment effect differences between DMTs. Comparative effectiveness studies to date have primarily focused on commonly used oral DMTs, specifically dimethyl fumarate (DMF), fingolimod (FTY) and teriflunomide. However, there are some publications reporting on head-to-head comparisons of oral versus infusion DMTs (e.g., FTY versus natalizumab [NTZ]) and multiple pairwise comparisons of injectable, oral and infusion therapies. DMT sequencing studies after discontinuation of DMF, FTY and NTZ have also been reported. As large datasets become increasingly available through multicenter studies and those 
using large, heterogeneous population-based databases that will comprise the newer infusion therapies, the MS literature will inevitably expand to include more comparative effectiveness studies investigating infusion versus infusion DMTs (e.g., NTZ vs ocrelizumab/rituximab).

\section{DMF, FTY \& teriflunomide}

A two-center, 36-month, PS weighting-adjusted analysis by Vollmer et al., showed similar effectiveness of DMF (n $=737)$ and FTY $(\mathrm{n}=535)$, measured via proportion with clinical relapses (odds ratio $[\mathrm{OR}]=1.27$; 95\% CI: 0.90 1.79), gadolinium-enhancing lesions $(\mathrm{OR}=1.25$; 95\% $\mathrm{CI}$ : $0.85-1.84)$ and new $\mathrm{T} 2$ lesions $(\mathrm{OR}=0.99 ; 95 \% \mathrm{CI}$ : $0.74-1.32$ ) with higher DMF discontinuations ( $\mathrm{OR}=1.81$; $95 \% \mathrm{CI}: 1.41-2.31$ ), largely driven by intolerance (OR = 1.63; 95\% CI: 1.18-1.73) [6]. To comment on DMT sequencing, patients who switched from DMF or FTY to highly effective therapy (HET; alemtuzumab, ocrelizumab, rituximab) showed decreased disease activity compared with those who switched to injectable/oral therapies (glatiramer acetate, IFN $\beta$, DMF, FTY and teriflunomide). Vollmer et al. also investigated MS sub-populations of interest through stratification (e.g., relapsing-remitting MS [RRMS], patients younger and older than 40 years of age, male and female patients, first-line and non-first-line users, those with and without baseline gadolinium-enhancing lesions, and direct switchers from NTZ) in a 24month DMF versus FTY study [7]. Overall, results showed comparable probability of absence of MS disease activity (measured via absence of clinical relapses and new MRI lesions) across all sub-groups, except for males $(\mathrm{OR}=0.58$; $\mathrm{p}=0.035)$ and first-line users $(\mathrm{OR}=0.67 ; \mathrm{p}=0.023)$ who experienced less risk with FTY treatment.

A 24-month comparative effectiveness study of DMF versus FTY by Fox et al. showed similar results [8]. The authors compared patient data across multiple studies with a matching-adjusted indirect method. Cross-trial differences were minimized by adjusting data from trials where individual patient data were known to match aggregate data from trials in which patient data were unknown. The matching-adjusted indirect comparison approach showed comparable efficacy of DMF and FTY measured via annualized relapse rate (ARR) $(R R=1.11$; 95\% CI: 0.88-1.40), though DMF was associated with better patient-reported outcomes. There were no significant differences in the percentages of patients with no evidence of disease activity (NEDA; RR $=0.92$; 95\% CI: $0.51-$ 1.64). Of note, confounding from unknown differences between trials and variations in trial length might have obscured treatment effect differences between the two groups.

A comparative effectiveness study by Boster $e$ al. utilized a health claims database to assess the real-world effectiveness of patients $(n=6372)$ newly-initiating DMF, IFN $\beta$, GA, teriflunomide and FTY [9]. Using a Poisson and negative binomial regression model, the adjusted incidence rate ratio (IRR) of relapses was comparable between DMF and FTY (IRR $=1.03 ; 95 \%$ CI: $0.88-1.21$ ), though significantly improved in DMF compared with IFN $\beta$ $(\mathrm{IRR}=1.27 ; 95 \%$ CI: $1.10-1.46)$, glatiramer acetate $(\mathrm{IRR}=1.34 ; 95 \% \mathrm{CI}: 1.17-1.53)$ and teriflunomide $(\mathrm{IRR}=$ 1.23; 95\% CI: $1.05-1.45)$ [10].

Ontaneda et al. used PS matching to compare the ARR of patients with MS ( $\mathrm{n}=20,311$ ) who switched from injectable therapy to either DMF, FTY or teriflunomide using a commercial health claims database [11]. Similar to previously reported DMF versus FTY studies, results of the current investigation showed a significant decrease in ARR for patients treated with DMF as compared with teriflunomide (rate ratio $[R R]=0.667 ; 95 \% \mathrm{CI}$ : 0.486-0.914) and comparable postindex ARR compared with FTY (RR = 1.07; 95\% CI: 0.861-1.328).

Laplaud et al. compared the effectiveness of teriflunomide $(\mathrm{n}=713)$ and DMF $(\mathrm{n}=1057)$ using inverse probability weighting [12]. At 24-month follow-up, teriflunomide and DMF demonstrated comparable clinical effectiveness measured via relapses and disability progression, but better MRI-based outcomes measured via new T2 lesions $(\mathrm{OR}=0.60 ; \mathrm{p}<0.001)$ [13]. Overall, this study provided Class III evidence that for patients with RRMS, teriflunomide and DMF demonstrated similar clinical effectiveness over 24 months of therapy. Another PS-adjusted analysis by Buron et al. compared DMF $(\mathrm{n}=767)$ versus teriflunomide $(\mathrm{n}=1469)$ in a real-world clinical setting [14]. The relapse RR for DMF versus teriflunomide favored DMF ( RR $=0.58$; 95\% CI: 0.46-0.73; $\mathrm{p}<0.001)$. Further, patients treated with DMF had a higher relapse-free survival proportion compared with those treated with teriflunomide at 48 months $(\mathrm{p}<0.05$ ). As opposed to Lauplad et al., this study provided Class II evidence that for patients with RRMS, DMF was more effective in preventing relapses compared with teriflunomide.

\section{FTY versus natalizumab}

A comparative effectiveness study by Lorscheider $e$ al. applied PS matching to evaluate FTY ( $\mathrm{n}=179)$ versus NTZ $(\mathrm{n}=179)$ as second-line therapy for RRMS patients who were nonresponders to first-line injectable DMTs [10]. 
Results showed that patients treated with NTZ had a lower risk of relapses compared with those treated with FTY (IRR $=0.5 ; 95 \%$ CI: 0.3-0.8). Further, NTZ patients had higher probability of Expanded Disability Status Scale (EDSS) improvement versus FTY patients (hazard ratio $[\mathrm{HR}]=1.8 ; 95 \%$ CI: 1.1-2.7). The authors concluded that NTZ was more effective in reducing relapse rates and disability progression as measured by the EDSS versus FTY.

A study by Baroncini et al. evaluated relapse data, EDSS scores and MRI data among RRMS patients treated with NTZ $(n=102)$ or FTY $(n=102)$ as second-line treatment using PS matching [15]. More patients discontinued NTZ compared with FTY, mostly due to safety concerns (33 vs $11 \%$; $p<0.001$ ). Patients treated with NTZ had a higher percentage of relapse-free status versus FTY (66 vs $80 \%$; $\mathrm{p}=0.015$ ), higher percentage of EDSS improvement ( 6 vs $15 \% ; \mathrm{p}=0.033$ ), lower percentage of MRI activity (38 vs $14 \% ; \mathrm{p}=0.001$ ) and higher percentage of NEDA ( $44 \mathrm{vs}$ $70 \% ; \mathrm{p}<0.001$ ). Similar to the Lorscheider et al. study, the investigators concluded that NTZ was superior to FTY across clinical and radiographic measures in RRMS patients nonresponding to first-line injectable therapies.

\section{Multiple pairwise comparisons investigating injectable, oral, \& infusion DMTs using a large heterogeneous MS population}

Using a large multicenter, multinational database, Kalincik et al. used PS matching to compare a number of DMTs. In 2017, the authors investigated alemtuzumab $(n=189)$ versus IFN $\beta(n=2155)$, FTY $(n=828)$ and NTZ ( $n$ = 1160) [16]. Patients treated with alemtuzumab showed lower ARR compared with IFN $\beta$ (0.19; 95\% CI: $0.14-$ 0.23 versus 0.53 ; $95 \%$ CI: $0.46-0.61)$ and FTY (0.15; $95 \%$ CI: $0.10-0.20$ versus $0.34 ; 95 \%$ CI: $0.26-0.41)$ and comparable ARR compared with NTZ (0.20; $95 \%$ CI: $0.14-0.26$ versus 0.19 ; $95 \%$ CI: $0.15-0.23)$. Alemtuzumab patients demonstrated similar probabilities of disability accumulation versus IFN $\beta$ (HR $=0.66$; 95\% CI: $0.36-$ 1.22), FTY (HR $=1.27 ; 95 \%$ CI: $0.60-2.70)$ and NTZ (HR $=0.81 ; 95 \%$ CI: 0.47-1.39). However, in terms of disability improvement, while alemtuzumab patients demonstrated similar probabilities compared with IFN $\beta$ and FTY, they had a lower probability versus NTZ (HR $=0.35$; 95\% CI: $0.20-0.59)$. Overall, patients treated with alemtuzumab demonstrated superior relapse rate reductions compared with IFN $\beta$ and FTY, while similar to that of NTZ. Results also demonstrated that NTZ appeared superior to alemtuzumab in allowing disability recovery.

In 2018, Kalincik et al. used PS matching to compare the effectiveness of cladribine $(\mathrm{n}=37)$ versus IFN $\beta$ $(\mathrm{n}=1940)$, FTY $(\mathrm{n}=1892)$ and NTZ $(\mathrm{n}=1410)$ [17]. Overall, patients treated with cladribine demonstrated a lower probability of relapses compared with IFN $\beta(\mathrm{p}=0.05)$, similar probability compared with FTY $(\mathrm{p}=0.31)$ and higher probability compared with NTZ $(\mathrm{p}=0.042)$. Further, patients treated with cladribine showed similar probability of disability accumulation compared with IFN $\beta(\mathrm{p}=0.37)$ and FTY $(\mathrm{p}=0.089)$, but demonstrated higher risk of disability compared with NTZ patients $(\mathrm{p}=0.021)$. However, cladribine patients had a higher probability of disability improvement compared with IFN $\beta(\mathrm{p}<0.001)$, FTY $(\mathrm{p}=0.0025)$ and NTZ $(\mathrm{p}<0.001)$.

In 2019, Kalincik et al. compared teriflunomide $(\mathrm{n}=614)$, DMF $(\mathrm{n}=782)$ and FTY $(\mathrm{n}=2332)$ over 2.5 years using PS matching [13]. Patients treated with FTY demonstrated lower ARR compared with both teriflunomide ( 0.18 versus $0.24 ; \mathrm{p}=0.05)$ and DMF $(0.20$ versus $0.26 ; \mathrm{p}=0.01)$; while ARR were similar between DMF and teriflunomide $(0.19$ versus $0.22 ; \mathrm{p}=0.55)$. There were no differences in disability accumulation nor improvement across all three DMTs.

\section{DMT sequencing following natalizumab discontinuation}

Recently, Hersh $e t$ al. published a two-center study using PS weighting that compared the effectiveness of switching from NTZ to a moderate-efficacy DMT (DMF $\mathrm{n}=130$; FTY $\mathrm{n}=140$ ) versus high-efficacy DMT (ocrelizumab $\mathrm{n}$ $=106$; rituximab $\mathrm{n}=17$, alemtuzumab $\mathrm{n}=7$ ) [18]. By 24 months post-NTZ, there were no significant differences in ARR across the two switched treatment paradigms (OR $=1.44 ; 95 \%$ CI: 0.69-1.59). However, patients who switched to moderate-efficacy DMT had more gadolinium-enhancing lesions $(\mathrm{OR}=3.62$; 95\% CI: 1.56-5.21), lower proportion with absence of disease activity $(\mathrm{OR}=0.41 ; 95 \% \mathrm{CI}: 0.21-0.71)$ and higher risk of disability progression $(\mathrm{p}=0.043)$. Similar results were observed across reasons for NTZ discontinuation (e.g., safety risks, breakthrough disease on NTZ), though they seemed to be driven more by those who switched from NTZ due to disease activity. The authors concluded that patients switching from NTZ to moderate-efficacy versus high-efficacy DMT were at higher risk of clinical and radiographic disease activity and progression by 24 months of follow-up.

Overall, these comparative effectiveness studies contributed to the MS literature by demonstrating DMT performance in real-world settings across a number of different databases (e.g., single- and two-center studies, health claims databases, large population-based databases). While they all differed in sample sizes, patient populations 
and outcome measures; they reported on similar conclusions that DMF and FTY have similar effectiveness and out-perform injectable therapies; and alemtuzumab and NTZ are highly efficacious therapies that demonstrate superiority over injectable and oral therapies in real-world practice. While FTY appears to be superior over teriflunomide, conflicting results were reported on the comparative effectiveness of DMF versus teriflunomide, for which more large, heterogeneous studies are warranted to further investigate this relationship. Such differences in reporting underscore the importance of designing robust observational studies that maximize data quality; incorporate a diverse patient population; are all-inclusive of relevant baseline demographic, clinical and paraclinical factors for statistical model building; and incorporate sensitivity analyses.

\section{Conclusion}

Clinical trial data, while valuable from a regulatory standpoint for the approval of new medications, have limited applications in clinical settings. Observational studies, on the other hand, can leverage large, heterogeneous data to derive real-world evidence for MS patients and providers. These studies use data from clinical practice that do not have the rigid constraints of clinical trials. As such, the results are more generalizable for the neurologist in the clinic and provide patients more realistic insights into how DMTs perform in a real-world setting. In the rapidly advancing landscape of novel neurotherapeutics for MS, it has become increasingly challenging, yet crucial, to compare the effectiveness and safety of DMTs. RCTs are cost- and time-prohibitive, making such trials impractical for providing these head-to-head comparisons. Comparative effectiveness studies using retrospective observational data are thus valuable for reporting on these treatment effect differences to inform decision-making in routine practice. Despite the pragmatic utility of observational studies, they are often prone to confounding by indication, and thus require methods to limit baseline imbalances in demographics and disease characteristics before direct comparisons are made. PS analysis is a unique statistical method that reduces the impact of indication bias, thereby approximating a randomized study design. With the utility of PS methodologies, real-world comparative effectiveness studies in MS can answer a wide array of clinically relevant questions with broad applicability, contributing to real-world evidence that not only support but complement RCTs.

Financial \& competing interests disclosure

C Hersh has received speaking and consulting fees from Genzyme, Genentech, EMD Serono, Novartis and Biogen. She has received research support paid to her institution from Biogen, Genentech and PCORI. L Hua has received speaking and consulting fees from Biogen, Genzyme, Genentech, Novartis, Celgene/BMS and EMD Serono. The authors have no other relevant affiliations or financial involvement with any organization or entity with a financial interest in or financial conflict with the subject matter or materials discussed in the manuscript apart from those disclosed.

Funded writing assistance from the Cleveland Clinic Lou Ruvo Center for Brain Health (CCLRCBH) was utilized in the production of this manuscript. Medical writer L Cordes, who is affiliated with $C C L R C B H$, assisted with manuscript preparation.

\section{Open access}

This work is licensed under the Attribution-NonCommercial-NoDerivatives 4.0 Unported License. To view a copy of this license, visit http://creativecommons.org/licenses/by-nc-nd/4.0/

\section{References}

1. Tintore M, Alexander M, Cosetllo K et al. The state of multiple sclerosis: current insight into the patient/health care provider relationship, treatment challenges, and satisfaction. Patient Prefer. Adherence 11, 33-45 (2017).

2. Trojano M, Tintore M, Montalban X et al. Treatment decisions in multiple sclerosis-insights from real-world observational studies. Nat. Rev. Neurol. 13(2), 105-118 (2017).

3. Rosenbaum P, Rubin D. The central role of the propensity score in observational studies for causal effects. Biometrika 70 , 41-55 (1983).

4. Rubin D. Using propensity scores to help design observational studies: application to the tobacco litigation. Health Serv. Outcomes Res. Methodol. 2, 169-188 (2001).

5. Rosenbaum P. Discussing hidden bias in observational studies. Ann. Intern. Med. 115, 901-955 (1991).

6. Vollmer B, Ontaneda D, Harris H et al. Comparative discontinuation, effectiveness, and switching practices of dimethyl fumarate and fingolimod at 36-month follow-up. J. Neurol. Sci. 407, 116498 (2019).

7. Vollmer B, Ontaneda D, Bandyopadhyay A et al. Discontinuation and comparative effectiveness of dimethyl fumarate and fingolimod in 2 centers. Neurol. Clin. Pract. 8(4), 292-301 (2018). 
8. Fox R, Chan A, Zhang A et al. Comparative effectiveness using a matching adjusted indirect comparison between delayed-release dimethyl fumarate and fingolimod for the treatment of multiple sclerosis. Curr. Med. Res. Opin. 33, 175-183 (2017).

9. Boster A, Nicholas J, Wu N et al. Comparative effectiveness research of disease modifying therapies for the management of multiple sclerosis: analysis of a large health insurance claims database. Neurol. Ther. 6(1), 91-102 (2017).

10. Lorscheider J, Benkert $\mathrm{P}$, Lienert $\mathrm{C}$ et al. Comparative analysis of natalizumab versus fingolimod as second-line treatment in relapsing-remitting multiple sclerosis. Mult. Scler. 24, 777-785 (2018).

11. Ontaneda D, Nicholas J, Carraro $\mathrm{M}$ et al. Comparative effectiveness of dimethyl fumarate versus fingolimod and teriflunomide among MS patients switching from first-generation therapies in the US. Mult. Scler. Relat. Disord. 27, 101-111 (2019).

12. Laplaud D, Casey R, Barbin L et al. Comparative effectiveness of teriflunomide vs dimethyl fumarate in multiple sclerosis. Neurology 93(7), e635-e646 (2019).

13. Kalincik T, Havrdova E, Horakova D et al. Comparison of fingolimod, dimethyl fumarate, and teriflunomide for multiple sclerosis. J. Neurol. Neurosurg. Psych. 90(4), 458-468 (2019).

14. Buron M, Chalmer T, Sellebjerg F et al. Comparative effectiveness of teriflunomide and dimethyl fumarate: a nationwide cohort study. Neurology 92, e1811-e1820 (2019).

15. Baroncini D, Ghezzi AP, Annovazzi P et al. Natalizumab versus fingolimod in patients with relapsing-remitting multiple sclerosis non-responding to first-line injectable therapies. Mult. Scler. 22, 1315-1326 (2016).

16. Kalincik T, Brown J, Robertson N et al. Treatment effectiveness of alemtuzumab compared with natalizumab, fingolimod, and interferon beta in relapsing-remitting multiple sclerosis: a cohort study. Lancet Neurol. 16, 271-281 (2017).

17. Kalincik T, Jokubaitis V, Spelman T et al. Cladribine versus fingolimod, natalizumab, and interferon beta for multiple sclerosis. Mult. Scler. 24, 1617-1626 (2018).

18. Hersh CM, Harris H, Conway D et al. Effect of switching from natalizumab to moderate- vs high-efficacy DMT in clinical practice. Neurol. Clin. Pract. doi:10.1212/CPJ.0000000000000809 (2020) (Epub ahead of print). 